\title{
Intelectuales tradicionales, educación de las mujeres y maternidad republicana en los albores del siglo XIX en Chile
}

\author{
Traditional intelectuals, women education and republican \\ motherhood at the start of the XIX century in Chile
}

\section{Edda HurTado P.}

Pontificia Universidad Católica de Valparaíso. Valparaíso, Chile

edda.hurtado@ucv.cl

\section{RESUMEN}

Este artículo se centra en el análisis de dos manuales pedagógicos publicados en los albores del siglo XIX chileno, en cuya elaboración y difusión se plasmó parte del ideario de los intelectuales tradicionales como gestores del itinerario cultural de la emergente república. En este periodo, la educación de las mujeres devino políticamente importante para la construcción de un imaginario nacional aunque pedagógicamente subalterna en relación al acceso a los conocimientos. Mercedes Marín del Solar y Rafael Minvielle configuraron un sujeto femenino bajo la lógica de las diferencias sexo-genéricas que aquí se revisan a partir de la noción de maternidad republicana.

Palabras clave: Literatura, educación, nación, escritores, manuales pedagógicos.

\section{ABSTRACT}

This article focuses on the analysis of two teaching manuals published at the turn of the 19th century, in whose production and dissemination, part of the thinking of traditional intelectuals was shaped as makers of the cultural itinerary of the emergent republic. At that period, women education became politically important for the shaping of national imaginary, but of secondary importance in terms of access to knowledge. Mercedes Marín del Solar and Rafael Minvielle built up a female subject 
following the logic of sex-gender differences, here reviewed as from the notion of republican motherhood.

Keywords: Literature, education, nation, writers, pedagogical manuals.

Recibido: 18.04.2011. Aceptado: 23.03.2012.

T a incipiente lógica nacionalista y la escritura de la historia literaria en los albores del periodo poscolonialista latinoamericano estuvieron determinadas por el rol desempeñado por los intelectuales fundacionales (De Marinis, 2006), quienes asumieron la función de gestores del itinerario de la cultura latinoamericana caracterizado por una mezcla de discursos heterogéneos que confluyeron en el diseño de los ideales de ciudadanía. Este proceso de formación identitaria nacional se expresó a través del interés en establecer el patrimonio cultural de la emergente nación, empeño en el cual no sólo se inventarió su herencia sino que también se inventó (Masiello, 1997). Los intelectuales fundacionales fueron intelectuales del (y para el) Estado-Nación, poniendo su tarea fundamentalmente al servicio de la construcción y la consolidación de la unidad política básica de la modernidad. Asimismo, pretendieron erigirse (y, a veces, tuvieron éxito en ello) en portavoces del conjunto de la sociedad o, al menos, de su principales fuerzas vivas. De tal forma, cumplieron un papel descollante en la elaboración de mitos de origen, historias nacionales y relatos identitarios fundamentales (De Marinis, 2006).

Coincidente con el romanticismo en las letras y el liberalismo en política, conservando rasgos diferenciadores respecto de la península ibérica, el interés por los desafíos que demandaba el diseño de la emergente nación no fue meramente especulativo, sino que estuvo directamente relacionado "con la necesidad de conocer, comprender y organizar la sociedad civil" y hasta las actividades más tradicionalmente desvinculadas de la funcionalidad práctica, "se articulaban a este proyecto de formar al ciudadano, al agente civil y civilizado de las nuevas repúblicas" (Osorio, 2000: 43). Literatura y nación a través del rol desempeñado por los letrados se instituyeron como fundamentos de la modernidad, que sin duda tuvo rasgos de periferia, pero no por ello menos significativa en tanto productora de nuevos sentidos y de resignificaciones del modelo europeo. Para la formación de este sentimiento incipiente de lo nacional, el desarrollo de las experiencias europeas se consideró traspasable toda vez que sus postulados parecían eficientes, conformándose así el entramado de una pedagogía de lo nacional (Thiesse, 2001), que 
daba por supuesto que este sentimiento debía enseñarse por la vía de una reformulación del concepto de civilidad que de manera incluyente comprometía a un gran espectro social. De este modo, la educación y la literatura se constituyeron como una pieza maestra en el dispositivo de la nación que no consistió sólo en la promoción controlada de la circulación de saberes relacionados con la enseñanza de la lengua y la gramática o la compleja problemática del estudio de la historia literaria, sino que los intentos de delimitación de un currículum nacional de enseñanza -proyecto que en Chile estuvo liderado por la Universidad de Chile y el Instituto Nacional, centros donde se forjaron y de donde irradiaron las directrices curriculares durante el siglo XIX- tuvieron como fundamento discursivo la necesidad de que la organización de estos saberes pensados y programados para ser difundidos a través del sistema educacional debían apuntar a modelar el cómo ser y cómo pensar nacionalmente; ello demandó una educación moral que se inscribió en las prescripciones curriculares, en los reglamentos de control disciplinario $^{1} \mathrm{y}$, en particular, a través de la enseñanza de la lengua y la literatura por medio de la selección de textos que los alumnos debían leer y memorizar. Igualmente, el aprendizaje de lo nacional y la construcción de experiencia nacionalmente valiosa (Goodson, 1995) no estuvieron exentos de peculiares interrelaciones de género ${ }^{2}$ (Masiello, 1997), instalando en la esfera pública la relación de las mujeres con la cultura y específicamente con la educación.

Intentando develar las particularidades de esta interrelación nos interesa analizar y relacionar dos manuales o dispositivos pedagógicos ${ }^{3}$ del siglo XIX pertenecientes a dos escritores nacionales: El libro de las madres i preceptoras.

1 El estudio de las humanidades clásicas también respondió a tecnologías disciplinarias (individualizantes e individualizadoras, centradas en los cuerpos de los individuos y destinadas a vigilarlos, controlarlos y adiestrarlos con el objeto de hacerlos dóciles y útiles) y reguladoras de la vida de los individuos (cuyo objetivo no sería tanto regular los cuerpos individuales cuanto el cuerpo social, las poblaciones). Ambas tecnologías se modularon de tal forma que se fortalecían para perfeccionar la vida individual y social, poniendo en marcha técnicas de racionalización y economía, tecnologías de un poder microfísico. Estas tecnologías involucraron la formación de nuevos saberes e instituciones: por una parte saberes destinados a jerarquizar, clasificar, vigilar y adiestrar los cuerpos y por otra, saberes destinados a la gestión global de la vida. Al respecto ver Stephen Hall (1992) y, también, el Reglamento para el Instituto Nacional decretado el 20 de diciembre de 1843 .

${ }^{2}$ Masiello examina "la relación entre mujeres y cultura desde comienzos del siglo XIX, periodo en que empieza a definirse un mundo poscolonial secularizado, hasta mediados de 1930, década en que culminó una larga experiencia de modernización que, en realidad, terminó en un fracaso. Con una ininterrumpida presencia de las tradiciones de las belles lettres desde 1830, las acciones femeninas en la cultura argentina son las más potentes de Latinoamérica (Masiello, 1997: 12).

${ }_{3}^{3}$ Empleamos el concepto de dispositivo pedagógico en tanto instrumento de prescripción curricular y disciplinar. 
Sobre la educación práctica de las mujeres, traducido del francés por Rafael Minvielle, recomendado por José Francisco Gana, decano de la Facultad de Filosofía y Humanidades de la Universidad de Chile para su difusión en las escuelas de la república, y el Plan de estudios para una niña ${ }^{4}$ de la poeta Mercedes Marín del Solar. Ambos manuales fueron elaborados en la década del cincuenta del siglo XIX en un momento en que el acceso de las mujeres a la educación formal se encontraba todavía en ciernes y la esfera literaria aún no estaba delimitada. La difusión de estos textos, especialmente de la traducción de Minvielle, da cuenta de la necesidad de institucionalizar los comportamientos de las mujeres considerados legítimos, a la vez que no sólo se debía explicitar las normas a las que referirse sino también disponer los dispositivos que permitieran su inculcación; en este sentido, la escuela, los libros y especialmente la difusión de estos manuales pretendían cumplir con este objetivo; "si bien los dispositivos se hallaban fuera de los textos y dependían de sus usos sociales, de sus lugares de utilización (la familia, la escuela), de su modo de apropiación (mediante una lectura individual y por medio de una palabra enseñante)", también estaban inscritos en el texto mismo que organizaba sus propias estrategias de persuasión y de inculcación (Chartier, 1994: 265 y ss.).

\section{EL ITINERARIO DE LOS TEXTOS: DIFUSIÓN, PUBLICACIÓN Y RESPALDO INSTITUCIONAL}

El libro de Rafael Minvielle corresponde a una traducción libre de una obra francesa premiada en la academia de Francia en 1845, cuya distribución en todas las escuelas primarias del país fue autorizada y promovida por la Facultad de Filosofía de la Universidad de Chile, otorgándole una legitimación institucional y consagrándolo oficial y legislativamente. El rol del traductor deja de manifiesto la intención de los intelectuales de hacer llegar las ideas que pregonaban a un público esperado y deseable e introducirlas en el plano de los hábitos y las costumbres. En sus más de 200 páginas, el contenido se divide en 32 capítulos con un prólogo en el que, a modo de informe, el decano José Francisco Gana destaca el valor de la obra en cuanto "necesario tratado de enseñanza", advirtiendo sobre la falta de libros "de este jénero" y considerándola como una obra "sólida i perfecta" a la vez que espera que con

\footnotetext{
${ }^{4}$ Este Plan nunca fue publicado y sólo es citado por Miguel Luis Amunátegui en dos oportunidades: En Doña Mercedes Marín del Solar (1867: 24-32) y en La alborada poética de Chile. Después del 18 de septiembre de 1810 (1892: 507-517).
} 
la ayuda de este texto la alumna "llegue a ser la mujer de la sociedad moderna”. El prólogo inscribe y despliega el deseo republicano de los escritoresestadistas de definir los lineamientos del nuevo Estado, que no excluían imaginar y representar las virtudes de la nacionalidad, interpelando a las mujeres como las mejores representantes de aquéllas. De acuerdo a la imaginación política de los hombres, la representación del concepto asociado a las mujeres en estos textos registraba imágenes que las identificaban con el caos y el desorden, lo que debía ser regulado en pos del proyecto civilizatorio.

En cuanto al Plan de estudios para una niña, redactado por la poeta Mercedes Marín del Solar, éste nunca fue publicado por su autora. Fue Miguel Luis Amunátegui quien en dos oportunidades y en obras de su autoría dio a la luz pública este plan redactado por Marín. La primera publicación de Amunátegui, titulada $D^{a}$. Mercedes Marín del Solar, dedicada a la vida y obra de la poeta, corresponde al año 1867 y el segundo momento en el cual el autor también transcribe este plan, corresponde al año 1892 en el libro La alborada poética en Chile. Después del 18 de septiembre de 1810. En este último libro, en el apartado dedicado a la poeta, Amunátegui señala que se trata de una transcripción íntegra de un apunte redactado "sin presunción de ningún jénero" y sostiene que esa obra ofrece el doble "interés de dar a conocer la idea que nuestra distinguida autora se había formado de la educación de la mujer, i de presentar un cuadro en que, sin quererlo ni saberlo, había bosquejado hasta cierto punto una pintura de sí misma". En ambos textos, Amunátegui destaca que se trata de "un plan en el cual la señora Marín ha reducido a teoría lo que una feliz inclinación natural le había enseñado a practicar ella misma” (Amunátegui, 1892: 24). En cuanto a la cantidad de páginas, la redacción no supera las diez.

El vínculo entre literatos y políticas educativas dirigidas a la educación de las niñas, las madres y las preceptoras fijó las pautas en un aspecto específico -la educación de las mujeres- que devino políticamente importante para la construcción de un imaginario nacional aunque pedagógicamente subalterna en relación a los conocimientos. El sujeto pedagógico femenino que ambos dispositivos elaboraron, si bien lo inscribían en el ámbito de la cultura letrada, confirmaba el hogar como el principal lugar pedagógico, delimitado bajo la lógica de las diferencias sexo-genéricas. El hogar se constituyó como el espacio principal de donde se esperaba que provinieran lineamientos más sistemáticos en torno a lo que las mujeres debían conocer y donde se les debería inculcar un saber vivir y un saber estar en sociedad. Este pasaje debía ser regulado en el hogar, dado que el acceso a los saberes escolarizados era aún inestable en términos de oportunidades para ellas. 
Los debates iniciales acerca de la importancia de la educación de las mujeres comienzan a darse a partir de la década del cincuenta del siglo XIX en Chile, instalándose una cuestión que no había sido prioridad ni preocupación estatal hasta entonces ${ }^{5}$. El escenario social y cultural presentaba rasgos controversiales en relación al acceso de las mujeres a la educación. Se trataba de un periodo en que el Instituto Nacional organizaba la educación secundaria de los varones bajo el denominado Plan Humanista de 1843, cuyo perfil tuvo una prioridad fundamental en cuanto estaba diseñado y orientado a la formación de "jóvenes chilenos -jóvenes de sexo masculino y pertenecientes a los grupos acomodados de la capital y ciudades más importantes" (Cruz, 2002), los que posteriormente participarían en los diversos ámbitos de la sociedad, fueran éstos políticos, económicos o administrativos; se trataba de la formación de los futuros hombres letrados que accederían al gobierno de la naciente república. El ingreso gradual de la mujer en la educación a lo largo del siglo XIX 6 comienza a demandar especial atención "en tanto su labor de reproducción social escapaba al control de los aparatos estatales destinados a la producción de sujetos pedagógicos y ciudadanos, y disputaba con la Iglesia Católica la formación de sujetos morales, es decir, de sujetos dotados de costumbres operantes en la vida cotidiana" (Poblete, 2003: 11-34).

\section{LA CASA COMO EL LUGAR PEDAGÓGICO POR EXCELENCIA}

La oposición binaria de lo público y lo privado vinculada al género -que situaba a las mujeres en el ámbito de lo privado y a los hombres en el de lo público- ha sido releída desde las implicaciones teóricas y políticas de los estudios culturales, dando paso a una importante resignificación del siglo XIX como el momento en que estos espacios comienzan a entrecruzarse claramente por medio del ejercicio de la escritura y la lectura en la casa que permitió a las mujeres ingresar progresivamente en el ámbito de lo público.

${ }^{5}$ La principal tribuna fue la prensa de la época y dos publicaciones que trataron el tema: De la Instrucción primaria en Chile, de los hermanos Miguel Luis y Gregorio Amunátegui, escrita en 1853 a raíz de un concurso convocado en 1853 por el Presidente de la República, Manuel Montt y De la educación popular, de Domingo Faustino Sarmiento. Estos autores proponían que debía hacerse una división entre escuelas de hombres y de mujeres, argumentando a favor de la instrucción primaria de las mujeres por sobre la de los hombres, porque sostenían que la mujer, por naturaleza, está más dispuesta que el hombre a transmitir los conocimientos que posee.

${ }^{6}$ La preocupación por la educación de las niñas ha sido ampliamente investigada desde la perspectiva de la historia de la educación en Chile y sólo como dato general -ya que no es el propósito de esta investigación- nos parece relevante señalar que hacia 1853 había 95 escuelas para niñas y 304 para niños (Egaña, Núnez y Salinas, 2003: 26). 
De este modo, el espacio doméstico sirvió a las mujeres para "desarrollar nuevos códigos de aprendizaje y para expandir la conversación pública a través de sus propias redes intradomésticas" (Masiello, 1997: 21). En este sentido, tanto Minvielle como Marín del Solar coinciden en fijar las bases de la formación de las niñas en el seno del hogar familiar, erigiéndose este espacio como principal referente para el aprendizaje de una civilidad en con alta influencia femenina en el hogar, que deriva de una doble pedagogía: la parental que refuerza el rol materno, y la imitación del ejemplo, que devuelve de nuevo a la imagen materna como modelo ideal "para inculcar, reconocer y reproducir el gesto justo. [En este sentido], los textos modulan sus preceptos en función de la posición social de la protagonista" (Chartier, 1994: 267), para lo cual se establecen los fundamentos morales y cristianos. En el plan de Mercedes Marín leemos:

Es preciso que una niña desde que principie a tener conocimiento consagre a Dios sus afectos, como las primicias de su alma. Para esto las madres tratarán de dar a sus hijas idea de Dios i de su bondad [...] dándoles edificantes ejemplos que se graben profundamente en sus almas i evitando todas las acciones i palabras que en lo mas mínimo pudieran manchar su inocencia (Amunátegui, 1892: 508).

Este fundamento moral y religioso resultaba complementario de la formación de las subjetividades ciudadanas masculinas, instaurando y legitimando culturalmente las claves diferenciadoras. En el marco del concepto de sociabilidad en lo que respecta a la educación, "en el siglo XIX existían dos vías para escribir el nuevo orden de la nación, de modo que el concepto operante de sociabilidad incluía tanto la letra (educación) como la práctica social, es decir, la costumbre" (Poblete, 2003: 15), situándose la educación de las mujeres como una necesidad compartida con otros sujetos y sectores subalternos en tanto "se apostaba a la necesidad de una reforma gradual de sus costumbres por la vía de una educación sabiamente administrada por los letrados (los hombres)"(16). Claramente, Rafael Minvielle permite demostrar estas afirmaciones en El libro de las madres... al desarrollar un plan basado en el ejercicio de la disciplina y el control, en tanto:

las mujeres nacen con particularidades tales como la debilidad, la pereza, la desidia, el egoísmo, la inclinación a la mentira, todas estas características que más tarde serán abono para vicios peores como la coquetería y las malas costumbres, $[\ldots]$ aquella niña que es embustera i dengosa será una 
despreciable coqueta, [indicios estos] i otros muchos más que merecen toda la atención de la madre (Minvielle, 1846:10).

Las virtudes de una mujer educada debían desarrollarse en base "al sentimiento religioso de su destino providencial, del amor al bien, del aprecio soberano a la verdad [...]". Según Minvielle, estos motivos elevados permitirían que con el estudio, la mujer afianzara "sus afecciones virtuosas" y así, no sacrificaría "su razón i su juicio a los juegos pueriles de la memoria i de la imajinación”, llegando a contar con el auxilio de todas sus facultades para inspirarse por sí misma y "mostrarse sucesivamente mujer de inteligencia, mujer de corazón, i también en los dias de prueba, la mujer fuerte de la escritura". Se trataba de insistir en la importancia de la educación de una mujer en relación a la influencia que se le reconocía en el ámbito privado "por su título de esposa i de madre" y si el mundo estaba dominado por el reino de la moral y el de la fuerza, se consideraba que este último debía siempre "estar en mano del hombre [y] el primer interés de la mujer será tratar de suavizarlo por todos los medios que estén a su alcance" (Minvielle, 1846: 18 y ss.). La lógica del discurso masculino organizado en oposiciones binarias encaminaba claramente la educación y la instrucción de la mujer.

En el seno de la familia, lugar pedagógico por excelencia y "abrigo de todo peligro moral", la niña estaría bajo "el ala protectora de la madre" y podría aprender los deberes "anexos a la condición de su sexo" (Minvielle, 1846: 22). No obstante, la escuela también presentaba sus ventajas para la educación de las niñas, ya que garantizaba -como primera regla- la disciplina a la que debían estar sujetas en tanto allí, la regulación del tiempo y el desarrollo de la virtud implicaban mecanismos disciplinarios destinados a controlar y adiestrar a las niñas con el objeto de hacerlas dóciles y útiles, y también implicaba mecanismos reguladores, en tanto la niña se inscribía a partir del ingreso a la escuela en una institución que contribuiría a su normalización. El disciplinamiento y la distribución homogénea y vigilada del tiempo se consideraban como un "correctivo al espíritu ardiente i caprichoso de la juventud", siendo la disciplina, "la que preserva de las fuertes excitaciones frecuentemente inevitables en el mundo" (Minvielle: 1846: 24). Mecanismos tales como la sujeción y el desarrollo de la virtud eran fundamentales en tanto regulación moral de la conducta de las niñas desde la infancia:

lo que ménos quieren comprender las niñas es la sujeción. Se imaginan que no están en este mundo sino para divertirse i gozar de todas sus comodidades, i como la vida está en perpetuo contraste con esta presunción llena de 
ignorancia i de simplicidad, es mui del caso sujetar desde un principio la juventud a la disciplina i a la obediencia (Minvielle, 1846: 32).

También se establecía que era necesario enseñar a las niñas que la virtud implicaba dudar de sí misma, criticando una civilidad puramente mundana y pagana; de este modo:

tendrá aquel pudor tímido que tan bien sienta a la inexperiencia; hablará poco, escuchará mucho, conocerá que necesita a los demás i estará por consiguiente libre de la presunción que notamos en numerosas caricaturas de mujeres i de hombres que hormiguean en los salones (Minvielle, 1846: $32)^{7}$.

En cuanto a la instrucción, en el texto de Minvielle se considera que se debían alcanzar todos los conocimientos útiles, pero en un grado relativo; las mujeres debían recibir sobre todo una educación "conveniente”, de acuerdo a su posición social y al destino al cual eran llamadas. El currículo de estudios era irrelevante, considerando a la lectura como un pasatiempo necesario. La representación de lo femenino demandante de disciplina y normalización surgían como una respuesta a la idea de una naturaleza femenina más próxima a la barbarie y que debía ser civilizada. En este sentido, la idea de civilización y barbarie se sostenía simbólicamente a través de las diferencias genéricas que aparecían como necesariamente complementarias en relación a la construcción de nación y a la familia como su núcleo fundamental. Al respecto, Francine Masiello sostiene que la unidad familiar en tanto microcosmos del Estado:

era invocada para proteger los intereses nacionales en la época poscolonial. Vinculados por lazos de sangre que les aseguraban una serie de afinidades comunes, los miembros de la familia eran llamados a proteger su hogar y a defender el espacio doméstico de la invasión. Por otra parte, esta estabilidad y orden domésticos cumplían una función policial mediante la cual los padres, que imponían paz en la familia, en última instancia tranquilizaban el clima de la nación (Masiello, 1997: 27).

${ }^{7}$ Según la prescripción, un medio eficaz de mantener a las niñas en este estado de pudor y recato es "no razonar mucho con ellas [...] porque las niñas particularmente gustan de argüir i discutir sobre todo, porque su respeto hacia sus superiores se altera mui luego, su confianza en sí misma va en aumento i afectan entónces un aire de importancia ridícula i quieren decidir sobre mil cuestiones fuera de su alcance". 
La apelación al lenguaje de lo familiar y por ende al lugar preponderante que la mujer debía jugar implicaba una jerarquía de la organización nacional al que Masiello se refiere como "el instrumento específico que articula el curso de la democracia latinoamericana en el siglo XIX en el debate sobre la construcción de la nación" (Masiello, 1997: 30). Al destacar las obligaciones de las mujeres en el hogar y sus cualidades, los intelectuales más notables crearon una imagen de la esposa y madre como modelo de la incipiente nación, impulsado "por una concepción de la política, la sociedad y la cultura vinculada al género [...] que se adecuaba a sus proyectos de Estado" (75). Lo que debería ser la familia republicana dio lugar a una leyenda de domesticidad que era necesaria para la consolidación de la familia y que, a la vez, se apropiaba de las virtudes femeninas en función del debate público.

\section{LA FAMILIA O EL NÚCLEO REPUBLICANO COMO LUGAR DE SUBVERSIÓN DE LA LETRADA}

La función desempeñada por las mujeres en el periodo de postindependencia definida como maternidad republicana se refiere al modo en que las mujeres ejercitaban su misión patriótica dentro del hogar (Masiello, 1997). El supuesto era que la vida de las mujeres estaba conformada primordialmente por necesidades y obligaciones familiares, junto a lo cual su responsabilidad era asegurar la virtud de la nación a través de su distinción biológica como madres. En otras palabras, se pensaba que las mujeres sólo podían dejar su marca en la sociedad a través del deber doméstico "es en el seno materno que reposa la civilización del mundo". El modelo familiar permitió consolidar posiciones políticas en el gobierno, reivindicándolo como un reflejo de sus valores cívicos y religiosos. Francine Masiello sostiene que este modelo operó como marco de referencia identificable para los intelectuales latinoamericanos, quienes al diseñar programas para las nuevas repúblicas reforzaron el rol tradicional de las mujeres:

La maternidad y lo doméstico desempeñaban una parte significativa en el programa de desarrollo nacional y la unidad familiar era concebida como un espacio para la formación de futuros ciudadanos [...] la mujer patriótica servía mejor a su país permaneciendo en el hogar (Masiello, 1997: 42).

En el Plan de estudios para educar a una niña de Mercedes Marín, el 
sujeto pedagógico femenino que allí se configura -tanto la madre como la hija- pertenece a este círculo en el que funciona el modelo de la familia republicana, inmerso en un ámbito de cultura letrada. La madre se configura como una mujer instruida en función de transmitir a sus hijos los valores cívicos. Si bien Mercedes Marín del Solar es una escritora considerada por Miguel Luis Amunátegui como la primera poeta postindependencia, el carácter subalterno de su escrito-borrador expone a su vez la subalternidad de la mujer escritora respecto del lugar que ocupaba en el espacio privado y también en el espacio de las emergentes letras nacionales. El plan para educar a una niña configura una destinataria de la oligarquía criolla, puesto que se asume que en el hogar hay libros, que la madre que la educa sabe leer y escribir, que puede corregir sus lecciones y donde se tiene conocimiento de lenguas extranjeras (sugiere dos por lo menos, enfatizando el estudio de francés). La niña destinataria de las sugerencias esbozadas por Mercedes Marín es representada en un microespacio doméstico que simbólicamente supone que sus miembros pertenecen a la cultura letrada a la que se le asigna un alto valor; sin embargo, los saberes están claramente diferenciados.

Marín se inscribe en el periodo de la naciente república, periodo de independencia política y de reorganización social; sin embargo, la clase dominante era conservadora en cuanto a la educación y destino de las niñas. La noción de orden, al igual que en el texto de Minvielle, es predominante en tanto pilar fundamental del ideario republicano que suponía que la familia debía conferir una apariencia de orden a la nueva sociedad. Sin duda que Marín convirtió su experiencia educacional -obtenida en el seno de su familia- en un modelo válido de educación. Desde su infancia estuvo en contacto con los más importantes políticos y literatos del periodo, especialmente con Andrés Bello, figura gravitante que reconoció el valor de su poesía, y a quien ella le escribía, configurándose como una especie de portavoz del sufrimiento de los otros. La poeta en algún momento se refirió a Bello como una especie de sombra protectora, paternal.

La subalternidad y su representación en la escritura la configuran como una escritora con un inestable posicionamiento en el espacio público, por lo tanto, si bien la propuesta pedagógica de Marín difiere relativamente de la propuesta de Minvielle, encontramos la confirmación de una "naturalización" de su propia vida configurada en la desigualdad genérica. No obstante, el Plan para educar a una niña es un texto más adelantado que el de Minvielle -aunque fueron escritos en el mismo periodo. Una de las afirmaciones más relevantes de este plan se refiere al latín como un conocimiento prescindible: "Una niña no tiene para qué estudiar latín". Probablemente, 
Mercedes Marín no estudió latín, sin olvidarnos que el dominio de esta disciplina era el salvoconducto para ser un ciudadano de primera categoría, a través de los estudios de secundaria realizados principalmente en el Instituto Nacional; pero también sabemos que adscribió a la generación de 1842 y Lastarria -que la menciona en sus Recuerdos Literarios- junto a otro importante grupo de liberales propugnó la abolición del estudio obligado del latín y su sustitución por el estudio de las lenguas vivas o modernas (Cruz, 2002). En este sentido, vemos a una Mercedes Marín afirmativa y liberal. Sin duda que el plan diseñado por Marín expresa un intento por demandar acceso al conocimiento. La disciplina que sugiere en relación a las materias y áreas que la niña debía conocer constituye el deseo de un modelo pedagógico que le permitiera refinar sus capacidades de abstracción, por ejemplo, a través de los conocimientos de geografía, de la explicación del sistema planetario y la iniciación en el estudio de la historia antigua:

Este precioso estudio debe hacerse de modo que produzca en el ánimo de la discípula una viva impresión de la magnificencia i hermosura de las obras del Creador, impresión cuyos resultados morales son incalculables [...] las reminiscencias históricas de los pueblos más célebres de la tierra inspiran a los niños el deseo de estudiar la historia (Amunátegui, 1892: 513).

Las mujeres letradas carecían de un marco teórico para la reflexión científica y filosófica, pero de algún modo, Marín sin proponérselo explícitamente establece un vínculo con saberes que estaban destinados a la formación humanista, exclusiva de los hombres que cursaban las humanidades. Si bien este plan presenta contradicciones en tanto fortalece un rol femenino circunscrito a la casa, favorece "a una madre instruida en el marco de un feminismo privado" que se inscribe como complementario de la estructura familiar tradicional. Una mujer instruida, tal como lo fue Marín del Solar, sería reconocida fundamentalmente en su rol de esposa y madre, pero se trata de una maternidad productivizada en la medida en que la obligación fundamental del rol femenino reside en la formación de futuros ciudadanos: "las jóvenes no pueden limitarse a aprender música, bellas artes y costura porque estas habilidades no sirven para que las mujeres se dediquen a un espectro de actividades vinculadas con la política y la filosofía. Por el bien social es necesario contar con una madre ilustrada" (Masiello, 1997: 88). 


\section{A MODO DE PROVISORIA CONCLUSIÓN}

En ambos textos se conjugan criterios que configuran una subjetividad femenina cuyos ejes centrales se inscribieron en el ámbito familiar, bajo la atenta mirada de quienes educaban o debían educar a las mujeres -madres o preceptoras- instaurando la importancia de la función maternal como un vector privilegiado en la transmisión de los valores elegidos para la formación de ciudadanos cristianos y republicanos. El libro de Minvielle, dado su carácter institucional, consolidaba férreamente el carácter moral y virtuoso, considerando que estaba destinado a las escuelas del país. Por su parte, la particularidad de haber sido escrito como un "borrador", sin intención -o capacidad- para ser publicado, permitió que Marín escribiera y prescribiera con una pluma más flexible y moderna los preceptos que -desde su propia experiencia- eran los fundamentales para la educación de una niña como futura saloniere.

La representación de lo femenino en ambos planes se presenta como un dispositivo funcional a un proyecto de una sociedad recién independizada que afrontaba la amenaza siempre presente del caos nacional, empleando el núcleo familiar y el reforzamiento de la figura materna y del orden doméstico como un lugar destinado a consolidar las bases de la emergente república. Del mismo modo, estos textos, en cuanto producción ideológica, creo que deberían ser revisados desde el revés del discurso, en cuanto a la función y el lugar social que ocuparon las mismas preceptoras, las institutrices o, desde una perspectiva social, poder historizar los modos y las prácticas sociales y culturales en que la mayoría de las mujeres analfabetas, sirvientas o campesinas se apropiaron de estos modelos de educación.

\section{REFERENCIAS}

Altamirano, Carlos (Dir.). 2008. Historia de los intelectuales en América Latina. Vol. I. La ciudad letrada, de la conquista al modernismo. Jorge Myers (ed.). Madrid: Katz Editores.

Amunátegui, Miguel Luis. 1867. Doña Mercedes Marín del Solar. Santiago: Imprenta de la República.

. 1892. La alborada poética en Chile. Después del 18 de septiembre de 1810. Santiago: Imprenta Nacional. 
Chartier, Roger. 1994. Libros, lectura y lectores en la Edad Moderna. Madrid: Alianza Editorial.

Cruz, Nicolás. 2002. El surgimiento de la educación secundaria pública en Chile, 1846-1876. El plan de estudio humanista. Santiago: Centro de Investigaciones Diego Barros Arana.

De Marinis, Pablo. 2006. "Los saberes de expertos y el poder de hacer y deshacer 'sociedad'", en Meeting of the Latin American Studies Association, San Juan, Puerto Rico, Marzo 15-18.

Egaña, María Loreto, Iván Núñez y Cecilia Salinas. 2003. La educación primaria en chile: 1860-1930. Una aventura de niñas y maestras. Santiago: LOM Ediciones/PIIE.

Goodson, Ivor. 1995. Historia del currículum. La construcción social de las disciplinas escolares. Barcelona: Ediciones Pomares-Corredor, S.A.

Hall, Stephen. 1992. Foucault y la Educación. Madrid: Morata.

Masiello, Francine.1997. Entre civilización y barbarie. Mujeres, nación y cultura literaria en la Argentina moderna. Argentina: Beatriz Viterbo Editora.

Minvielle, Rafael. 1846. El libro de las madres y las preceptoras. Sobre la educación práctica de las mujeres. Santiago: Imprenta de los Tribunales.

Osorio, Nelson. 2000. Las letras hispanoamericanas en el siglo XIX. Santiago de Chile: Coedición Universidad de Alicante y Universidad de Santiago.

Poblete, Juan. 2003. Literatura chilena del siglo XIX. Entre públicos lectores y figuras autoriales. Santiago: Editorial Cuarto Propio.

Thiesse, Anne Marie. 2001. La créations des identités nationales. Europe XVIIIe - Xxe siècle. Paris: Editions du Seuil. 